\title{
Special Section Guest Editorial: Laser Damage V
}

\author{
Vitaly E. Gruzdev ${ }^{a}$ and Jonathan W. Arenberg ${ }^{b}$ \\ ${ }^{a}$ University of New Mexico, Department of Physics and Astronomy, Albuquerque, \\ New Mexico, United States \\ ${ }^{b}$ Northrop Grumman Space Systems, Redondo Beach, California, United States
}

This special section on laser damage, Laser Damage V, the fifth in a series of special sections in Optical Engineering (OE), is focused on the diverse aspects of laser-induced damage (LID) of optical materials and components. Previous special sections were published in December 2012, December 2014, January 2017, and December 2018.

The field of LID of optical materials considers a wide range of phenomena, processes, and effects attributed to the high-power, high-energy, and high-intensity laser-material interactions that irreversibly and permanently modify the optical properties of the materials. Examples of these permanent changes in materials are an increase of scattering or reduction of transmittance. LID has been a fundamental limitation to the performance of lasers and laser systems from the earliest days of the laser epoch to the present. It is a technical problem that is of global interest to the laser community. The major strategic aims of the research efforts of the LID community are to properly characterize threshold of LID and the identification approaches that increase the LID threshold. Over 50 years of research in LID of optical materials has resulted in tremendous progress in terms of understanding the fundamental mechanisms of LID and orders-of-magnitude improvements of LID threshold of laser optics. Despite the significant advances made, the problem of LID has not been generally solved.

Since LID is a fundamental limitation to the advancement of the field, the global laser community is keenly interested in all topics related to laser damage. A major motivation of the enduring interest in LID is the continuous development of novel laser systems. These new systems and sources probe new ranges of laser parameters, placing ever greater demands on their materials, optics, and coatings. Current trends creating these new challenges, such as generation of subfemtosecond (attosecond) pulses, reduction of laser wavelength towards deep-ultraviolet and $\mathrm{x}$ ray ranges of the spectrum, extension of wavelength of high-power laser systems towards midinfrared range, and continuous increase of peak power of laser systems well above tera-watt level. The development and operation of large-scale peta-watt laser facilities operating, such as the National Ignition Facility and Omega Laser Facilities (USA), ELI Beamlines Facility (European Union), Mega Joule Laser facility (France), SG-III facility (China), and ILE/ Osaka University Large-Scale Laser facility (Japan), continuously supports and drives the need for advancement in LID research worldwide. In addition to the interest in LID studies generated by new systems, the vibrant global market in laser optics that drives ever greater quality and lower prices also places demands on the damage community.

As with all of OE's special sections on laser damage, this special section is inspired by the community associated with the annual SPIE Laser Damage Symposium, also known as the Boulder Damage Symposium. The 51st meeting of the symposium was held as a virtual conference in September 2020 by SPIE. In spite of the significant challenges resulting from the COVID-19 pandemic, that virtual meeting successfully continued the line of conferences first organized by Arthur Guenther and Alex Glass in 1969. Initially conceived as a small topical meeting to rapidly resolve some "small issues" associated with the failure of optical components in high-power lasers due to damage. Those "small issues" have matured into a significant and expanding international field of research and the subject of the annual meetings. Intensive development of high-power lasers and applications in Southeast Asia have motivated the establishment of a Pacific Rim Laser Damage (PLD) conference, first held in 2009 by Shanghai Institute of Optics and Mechanics (China) in cooperation with SPIE. Another indicator of the active

(C) 2021 Society of Photo-Optical Instrumentation Engineers (SPIE) 
growth of interest in the field is the fact that many SPIE and OSA conferences on high-power lasers now include sessions devoted to LID and related issues. The recent OSA Laser Congress featured a session dedicated to LID.

The decision to prepare this special section was motivated by the enduring interest in LID issues worldwide and by the pivotal success of the previous four special sections on laser damage. Since the previous four special sections on laser damage in OE, significant results have been presented at both LD and PLD meetings that deserve publication in a peer-reviewed journal. This Special Section on Laser Damage V contains nine papers on the major aspects of LID. The reduced number of publications as compared to the previous four special sections is attributed to the general impact of the COVID-19 pandemic on the research community worldwide an impact felt by the contributing authors and the editors.

Of the nine papers published in this special section, five are directly relevant to laser-surface interactions and influence of surface contamination on LID. The paper by Kafka et al. is focused on the study of the fundamental mechanisms of surface damage induced by contamination particles on a high reflector. It is shown that depending on nature and geometry of contaminants, the LID may result from interference of incident and reflected laser radiation, absorption by the particles, and combined effects from interference and nonlinear absorption. Those mechanisms were perfectly illustrated by experiments with artificially produced contamination particles that mimic real contaminants. The damage issues attributed to surface contamination are also considered by El Reaidy and Gallais. The focus of this work is on the contamination induced by high-average-power ultrashort pulses delivered at $\mathrm{MHz}$ repetition rates on surface of multilayer high reflector exposed to ambient atmosphere. Dynamics of growth, morphology, and composition of the contamination are characterized. The authors suggest the formation of nanometersized layers of carbonate compounds within the laser spot by evidence of contribution form thermal effects. Approaches to mitigate that effect are discussed. The contribution of Cross and Carr considers the generation of high-fluence precursors on the surface of fused-silica substrates. The issue of damage precursors is one of the fundamental challenges for operation of the petawatt laser facilities and continuously receives significant attention. This manuscript reports on the continuing progress of the research team from Lawrence Livermore National Laboratory. The influence of surface defects on LID of KDP crystals continues to be a problem of significant interest to the LID community. The reason for this ongoing interest is that KDP is commonly utilized for frequency conversion in high-power laser systems. The report from Geng et al. is devoted to studies of damage initiation by interaction of defects on KDP surfaces with ultraviolet nanosecond laser pulses. By use of various defect-characterization methods, in-situ damage tests, and pump-probe shadowgraph imaging, two types of participating defects are identified: the surface defects associated with lower LID threholds, and sub-surface (bulk) defects attributed to higher thresholds. Van Woerkom et al. consider fundamental aspects of surface modification by high-power picosecond laser pulses that result in ablation of metal and semiconductor surfaces. The focus of this paper is on the effects of transverse structure on ablation morphology as well as ablation efficiency.

LID of thin films is the theme of the remainder of the contributed papers for this special section. The contribution by Wang and Cox considers damage of anti-reflection (AR) coatings on calcium fluoride windows induced by nanosecond pulses of ultraviolet ArF laser. The AR coatings were integrated with protective coatings to enhance their damage resistance while maintaining low reflectivity at laser wavelength. The results come in spite of the usual result that LID thresholds of AR coatings are usually lower than those of substrates. This paper identifies twophoton absorption in the $\mathrm{CaF}_{2}$ substrate as the major LID mechanism for this case. Kozlov et al. report on the long-term stability of multilayer dielectric gratings utilized in compressors of 10and 100-picosecond laser pulses at the OMEGA laser facility at the Laboratory for Laser Energetics. These gratings must survive multiple laser pulses, and stability of their damage performance is of keen interest for high-power systems. The report includes data accumulated over 10 years of routine damage tests which strongly suggest the long-term stability clearly depends on pulse width. Lamaignere et al. present round-robin measurements of LID threshold with subpicosecond laser pulses in two optical single-layer optical coatings performed at five testing facilities. Since the LID by sub-picosecond laser pulses is frequently characterized by an abrupt switch of LID probability from zero to $100 \%$ within a narrow range of laser fluence, the samples 
were expected to demonstrate quite similar LID thresholds. The objective of this research was to check influence of parameters of the testing facilities (e. g., environment, damage detection methods, sample homogeneity, temporal and spatial profiles of testing lasers) on results of LID threshold measurements. The paper analyzes reasons for the significant deviations of the thresholds obtained at the participating facilities. This paper has implications for the development of standards now being considered domestically and internationally. The paper of Liao et al. is focused on the special features of fluence distribution over $\mathrm{N}$ laser shots on a surfaces of optics of the National Ignition Facility. Generation and support of homogeneous fluence distribution is one of the major approaches considered to improve damage performance of optics surfaces via suppression of hot spots. This paper concludes that the fluence variations at a given point of optics surface result from both fluence fluctuations and pointing jitter of the laser. Based on those results, approaches to mitigate the local fluence fluctuations on surfaces of large-scale optics are discussed.

The papers collected in this, the fifth special section on laser damage, continue to show the vibrant research done by researchers around the world. The editors hope that the continuing and newly emerging research developments in the field of laser-induced damage and optical materials for high-power lasers represented by these papers will be of benefit to the readers of Optical Engineering and researchers from multiple related areas. The editors also feel there is value in bringing together these papers in this special section so that they might be considered jointly.

Vitaly E. Gruzdev received an MS in optical systems and devices from the Institute of Fine Mechanics and Optics (ITMO University) in St. Petersburg, Russia, in 1994, and a PhD from the Federal Research Center "S. I. Vavilov State Optical Institute" in St. Petersburg, Russia, in 2000 in the field of optics. Since 1994, he has been doing research in the field of fundamental mechanisms and effects of high-intensity laser-solid interactions and laser damage in transparent solids. He was with the Laboratory of Surface Photophysics of Dr. M. Libenson and then with the Photophysics Laboratory of Dr. A.M. Bonch-Bruevich at S.I. Vavilov State Optical Institute (Russia). He was a visiting researcher with the group of Prof. Dr. D. von der Linde from January 2001 to December 2003. In November 2005 he joined the Center for Ultrafast Ultraintense Lasers at the College of Engineering, University of Missouri. Since September 2018, he has been a research associate professor with the Department of Physics and Astronomy of the University of New Mexico in Albuquerque, NM. Since 2009, he has been a co-chair of the SPIE Laser Damage Symposium.

Jonathan W. Arenberg received his BS in physics and an MS and $\mathrm{PhD}$ in engineering, all from the University of California, Los Angeles. He has been an active and contributing member of the LID community since 1986. His major research interests center around measurement methods and standards. He is on the US national and ISO committees drafting standards. He is the international program committee for the SPIE Laser Damage Symposium and an SPIE Fellow. 